\title{
Interaction of Transfusion and Iron Chelation in Thalassemias
}

John Porter and Maciej Garbowski

University College London Cancer Institute

Sent to Hematology/Oncology Clinics of North America

\begin{abstract}
Transfusion combined with iron chelation has been the paradigm for management of severe $\beta$ thalassemia syndromes for over 6 decades; during which time both mortality from cardiosiderosis and quality of life have steadily improved. Transfusion aims at ameliorating anemia and suppressing erythron expansion while not transfusing more iron than is necessary. Blood transfusion inevitably leads to iron overload with secondary mal-distribution of body iron. Overly intense transfusion regimens, as marked by low soluble transferrin receptors, not only increase the rate of body iron accumulation but also suppress endogenous erythropoiesis. Increased transfusion also decreases the ratio of chelation derived from red cell metabolism relative to chelation from hepatic stores. Conversely low transfusion rates increase endogenous erythropoiesis with the generation of iron-free transferrin that can then bind NTBI species otherwise destined for myocardium or endocrine tissues. When the transfusion rate exceeds the clearance of transferrin iron by the erythron, the risk of cardiosiderosis is increased with an odds ratio of 48. Cardiosiderotic risk is also increased when total body iron, as determined by LIC was not controlled. Optimal chelation dosing and regimen should aim to control body iron by balancing the transfusional iron-loading rate with the chelation-induced excretion and also providing 24-hour protection from plasma NTBI. Once cardiosiderosis has developed, its clearance even with intensified chelation often lags behind hepatic clearance. A systematic reappraisal of the impact of transfusion practices, particularly pre-transfusion Hb values, on the success in preventing cardiosiderosis and chelation response is indicated.
\end{abstract}




\section{Introduction}

The benefits of both transfusion and chelation when used together are well established for transfusiondependent thalassemias. With the advent of newer chelation regimens and monitoring techniques, and in the light of recent data linking low erythron activity to increased risk of cardiosiderosis, a reappraisal of the optimal balance between transfusion rates and chelation may be warranted. In this paper, current transfusion and chelation practices are critically reviewed with particular emphasis on how transfusion strategy affects iron distribution and the mechanisms through which this interaction occurs. The implications of this interaction for chelation strategy are also examined.

\section{Objectives of transfusion in thalassemias}

Blood transfusion in thalassemia aims to correct anemia so that physical and cognitive performances are close to healthy individuals, while preventing harmful or potentially disfiguring expansion of the erythron and not transfusing more blood and hence iron than necessary. Evidence for optimal hemoglobin $(\mathrm{Hb})$ to achieve these goals was initially contradictory: some studies showed transfusion requirements remained constant at mean transfusion $\mathrm{Hb}$ levels of $10-14 \mathrm{~g} / \mathrm{dL}$ (equivalent to pretransfusion $\mathrm{Hb} 8-12 \mathrm{~g} / \mathrm{dL})^{1,2}$, while others showed that transfusion requirements were directly proportional to the mean $\mathrm{Hb}^{3,4}$. In a later study, transfusion requirement was measured in the same patients under two transfusion regimens ${ }^{5}$ : those with mean pre-transfusion $\mathrm{Hb}$ of $11.3 \mathrm{~g} / \mathrm{dL}$ had mean annual transfusion of $137 \mathrm{~mL} / \mathrm{kg}$ but when the mean was subsequently lowered to $\mathrm{Hb} 9.4 \mathrm{~g} / \mathrm{dl}$, annual consumption decreased to $104 \mathrm{~mL} / \mathrm{kg} / \mathrm{L}$ and ferritin values fell.

Current guidelines recommend pre-transfusion $\mathrm{Hb}$ values of $9.5-10.5 \mathrm{~g} / \mathrm{dl}$, thus keeping the mean $\mathrm{Hb}$ at about $12 \mathrm{~g} / \mathrm{dl}$ with a post-transfusion $\mathrm{Hb}$ not above $14 \mathrm{~g} / \mathrm{dl}^{6}$. This 'sweet spot' for balancing considerations of iron loading and correction of anemia and ineffective erythropoiesis (IE) is based on a study of Italian patients ${ }^{5}$ and may not be applicable to patients with different levels of effective erythropoiesis (see below). Under certain circumstances, higher Hb levels may be appropriate, for example, when some patients experience low back pain at $\mathrm{Hb}<10-11 \mathrm{~g} / \mathrm{dl}$, or when the spleen size is expanding, or during pregnancy. The recommended frequency of transfusion every 2-4 weeks has been determined to some extent by the convenience to patients and the availability of blood in some regions. Mathematical modeling suggested pre-transfusion hemoglobin of $9 \mathrm{~g} / \mathrm{dL}$ with transfusions every two weeks rather than every four weeks would reduce requirements by $20 \%$ but no measurable effect was seen in a study comparing 3- or 4-week intervals ${ }^{4}$.

Some populations are managed on lower pre-transfusion $\mathrm{Hb}$ values: for example, in 464 Egyptian TDT patients aged 10 months to 31 years $^{7}$, the mean pre-transfusion $\mathrm{Hb}$ was $5.7 \mathrm{~g} / \mathrm{dl}$. In another study, the $\mathrm{Hb}$ values were significantly lower in patients from Cairo, Egypt (6.9g/dl) than from Ismir, Turkey ( $\mathrm{Hb} 8.9 \mathrm{~g} / \mathrm{dl}$, Y. Aydinok, personal communication). We suggest below that this variation in practice may account for differences in the proportion of patients with cardiosiderosis (see below).

A further consideration is whether and when to start transfusion, particularly for milder syndromes. Many patients with milder phenotypes may not require transfusion in the first few years of life but as $\mathrm{Hb}$ values fall, particularly if there is failure to thrive, transfusion may become necessary. Guidelines generally suggest $\mathrm{Hb}$ values repeatedly $<7 \mathrm{~g} / \mathrm{dl}$ are suitable to begin transfusion but this approach may not be universally applicable, particularly in E $\beta$-thalassemia syndromes, where the oxygen dissociation curve is right-shifted relative to $\beta$-thalassemia syndromes. For example in a study where $109 \mathrm{E} \beta$-thalassemia patients from Sri Lanka were followed for 5 years, the un-transfused group had $\mathrm{Hb}$ levels of $6.1 \mathrm{~g} / \mathrm{dl}$ and, based on performance status, did not require starting transfusion, while in a second group with mean $\mathrm{Hb}$ values of $7.0 \mathrm{~g} / \mathrm{dl}, 40 \%$ were able to stop transfusion without deleterious effects, despite the low $\mathrm{Hb}_{\text {values }}{ }^{8}$. These findings suggest that $\mathrm{E} \beta$-thalassemia can be often managed without transfusion, even with low hemoglobin levels.

\section{Body iron content and impact of transfusion.}

Thalassemia patients who are anemic but not receiving regular blood transfusion, so called nontransfusion-dependent thalassemia (NTDT), absorb increased quantities of dietary iron, due to inappropriately low hepcidin relative to iron stores, that may not be balanced by insensible iron losses, 
so that iron overload gradually develops ${ }^{9}$. Excess iron absorption depends on the degree of IE, the extent of erythroid expansion and the severity of anemia. Iron absorption rates determined with radioisotope markers, showing absorption of 5 times that of healthy controls were reported in $\beta$-thalassemia intermedia (range 3-10 times) ${ }^{10}$. This was similar in E $\beta$-thalassemia, where studies also showed that iron absorption correlated with plasma iron turnover, transferrin saturation, and liver iron concentration $^{11}$. Iron absorption over 1 year has now been determined in larger populations, using MRI to measure changes in LIC $^{12}$ and hence calculate net iron absorption ${ }^{13}$. LIC and serum ferritin increased from baseline by $0.38 \mathrm{mgFe} / \mathrm{g}$ dry weight and $115 \mathrm{ng} / \mathrm{mL}$, respectively. This increase in LIC, is equivalent to an average iron accumulation rate of $0.011 \mathrm{mg} / \mathrm{kg} / \mathrm{day}^{12}$. Increased iron absorption is currently thought to result from inhibition of hepatic hepcidin synthesis by bone-marrow-derived factors associated with IE and leading to inappropriately low hepcidin levels relative to iron stores ${ }^{9}$. Implicated factors have included growth differentiation factor-15 (GDF-15) in humans ${ }^{14}$ and, more recently, erythroferrone in mice ${ }^{15}$. GDF-11 has also been identified as a factor associated with IE in a murine thalassemia model ${ }^{16}$. Iron overload in NTDT correlates with erythron expansion biomarkers (sTfR, GDF-15, NRBCs), particularly in un-transfused, such as Hemoglobin H, patients ${ }^{9}$. Plasma hepcidin correlates inversely with TfSat, NTBI and LPI $^{9}$, consistent with a link between iron absorption and depressed hepcidin levels. Hepcidin/SF ratios were also low, consistent with hepcidin suppression relative to iron overload ${ }^{9}$. Increased NTBI and, by implication, risk of extra-hepatic iron distribution was more likely in previously transfused, splenectomized and iron-overloaded NTDT patients with TfSat $>70 \%{ }^{9}$.

The iron-loading rate from blood transfusion in TDT is about 10 times the rate seen in NTDT or 20$35 \mathrm{mg} /$ day in a $70 \mathrm{~kg}$ adult ${ }^{17}$. This is because of the high iron content in a unit of blood: a unit of red cells, processed from $420 \mathrm{~mL}$ of donor blood, contains approximately $200 \mathrm{mg}$ of iron (or $0.47 \mathrm{mg} / \mathrm{mL}$ of whole donor blood equivalent to $1.16 \mathrm{mg} / \mathrm{mL}$ of pure red cells). The mean loading rate is approximately $0.4 \mathrm{mg} / \mathrm{kg} /$ day $^{17}$ equivalent to $28 \mathrm{mg} /$ day of iron in $70 \mathrm{~kg}$ adult. About $20 \%$ of patients receive $<0.3 \mathrm{mg} / \mathrm{kg} / \mathrm{day}$, about $60 \%$ receive $0.3-0.5 \mathrm{mg} / \mathrm{kg} /$ day, and a further $20 \%>0.5 \mathrm{mg} / \mathrm{kg} / \mathrm{day}$ of iron. Without chelation therapy, LIC reaches $15 \mathrm{mg} /$ dry weight after less than 5 years of blood transfusion. Average transfusion requirements are somewhat higher in un-splenectomized $(0.43 \mathrm{mg} / \mathrm{kg} / \mathrm{day})$ TDT patients compared with splenectomized patients $(0.33 \mathrm{mg} / \mathrm{kg} / \mathrm{day})^{17}$. Thus splenectomized patient receive the equivalent of $300 \mathrm{~mL}$ less of whole blood per $\mathrm{kg}$ per annum decreasing the annual iron loading by $39 \%$. This is not sufficient reason to recommend splenectomy due the high complication rates and because modern chelation regimes can generally keep pace with this difference in iron accumulation. In patients who develop splenic enlargement, hypertransfusion can often diminish spleen size without the need for splenectomy ${ }^{18}$.

\section{Mechanisms of body iron distribution in thalassaemias}

In the absence of blood transfusion, excess absorbed iron is found in the peri-portal hepatocytes in $\mathrm{NTDT}^{19}$ and extra-hepatic iron distribution is very rare ${ }^{20,21}$. However with blood transfusion in TDT, iron accumulates initially in the macrophage system after erythrophagocytosis of transfused red cells. Red cell heme is catabolized here by haemoxygenase with iron rapidly released through ferroportin channels for extracellular binding to transferrin. Alternatively, when hepcidin binding to membrane ferroportin degrades this molecule, more iron can be retained within macrophages as storage iron (ferritin or hemosiderin). The macrophage system is considered capable of storing about $10 \mathrm{~g}$ of iron (or about 50 units of transfused blood).

Once the macrophage system is replete, increasing proportions of storage iron are delivered to hepatocytes though diferric transferrin and/or through plasma iron species occurring when transferrin saturation exceeds about $75 \%$, the so-called plasma non-transferrin-bound iron, NTBI. Unlike transferrin-bound iron, which is targeted through transferrin receptors to the erythron or to hepatocytes, NTBI species are taken into endocrine system and myocardium, in addition to hepatocytes, probably though LVDCC channels into myocardium ${ }^{22}$ and possibly, also though Zip14 in the endocrine system ${ }^{23}$. The cells known to take up NTBI are similar to those tissues susceptible to the effects of transfusional iron overload (see below). Clinical conditions associated with high plasma NTBI, such as TDT and DBA, are associated with high NTBI levels. Conversely, conditions with low NTBI levels even where severe iron overload co-exists, such of NTDT and sickle cell disease, have a low propensity to extra-hepatic iron distribution ${ }^{24}$. Levels of NTBI are affected by several independent factors $^{24}$, being raised by iron overload, but are also raised when erythropoiesis is relatively inactive, 
such as in myelo-ablative chemotherapy, due to decreased clearance of transferrin-iron by the erythron $^{25}$ or when erythropoiesis is constitutively inactive, such as in $\mathrm{DBA}^{24}$ (Figure 1).

Cellular iron uptake from NTBI is not only dependent on plasma levels but also on its speciation ${ }^{26}$. NTBI is heterogeneous consisting largely of iron-citrate species where the molecular weight of the iron-citrate complex is determined by the ratio of constant plasma citrate concentrations to the variable concentrations of $\mathrm{NTBI}^{27}$. Recent work suggests that the makeup of iron citrate species (their speciation) has a key impact on the iron uptake into tissues such as myocardium ${ }^{26}$. Thus iron uptake in HL-1 cardiomyocytes and generation of intracellular ROS occured most rapidly when citrate exceeded iron(III) by more than 100:1. Such conditions favor kinetically labile monomer (monoferric) rather than oligomer citrate species. These same species are those detected by the LPI assay which measures the redox-active compnent of $\mathrm{NTBI}^{28}$. Monoferric species also bind most rapidly to apotransferrin, which therefore readily inhibits cellular iron uptake of these NTBI species ${ }^{26}$. Thus apotransferrin generated by high transferrin-iron utilization in an active bone marrow can inhibit NTBI tissue uptake by rapidly binding monoferric-citrate species (see below). Therefore the activity of the erythron is critical to NTBI speciation and hence body iron distribution. This expains why patients with iron overload but with little utilization of transferrin-iron, such as DBA or heavily hypertransfused TDT patients, may be particularly susceptible to extra-hepatic iron distribution.

The clinical consequences of this iron distribution, including cardiomyopathy, endocrinopathy, and bacterial infections, have been recently described and reviewed by the senior author ${ }^{29}$. The generation of ROS by iron that redox-cycles between iron(II) and iron(III) is the major driver of oxidative tissue damage and iron chelation therapy has had a major impact on the frequency of these complications $\mathrm{s}^{30}$.

\section{Effect of blood transfusion regimen on iron distribution}

\section{a) Effect of cumulative blood transfusion on iron distribution}

In the absence of regular transfusion in NTDT, a lack of cardiosiderosis has been consistently reported $^{20,21}$. However, once transfusion begins in TDT, there is a clear link between the risk of myocardial iron accumulation and the cumulative volume of blood transfused. This was clear in the pre-chelation era $^{31}$ where interpretation was not muddied by chelation altering the relationship between liver and cardiac iron. Post mortem data on multi-transfused patients with a variety anemias showed that $100 \%$ of patients had evidence of increased cardiosiderosis after 200 transfused units ${ }^{31}$. The proportion fell as the number of transfused units decreased; thus $60 \%$ of patients had increased heart iron after 100 units, but only $10 \%$ after 25-50 units ${ }^{31}$. Early MRI studies also reported that myocardial iron increased in myelodysplastic patients with the number of transfused blood units and with LIC $^{32}$. In studies failing to show such a link ${ }^{33}$, interpretation has been confounded by recent chelation therapy, which perturbs the apparent relationship between liver and heart iron due to more rapid removal of liver than heart iron. It is important to examine trends of heart and liver iron over time, where a clear relationship between these has been shown in chelated patients with heart changes following those in the liver ${ }^{34}$.

\section{b) Effect of intensity of transfusion regimen on iron accumulation and iron distribution}

The intensity of blood transfusion can be represented by the pre-transfusion $\mathrm{Hb}$ combined with the frequency of transfusion, which contribute to mean $\mathrm{Hb}$. The higher this is, the greater the suppression of endogenous erythropoiesis ${ }^{5}$. The most robustly studied marker of extra-hepatic iron is myocardial $\mathrm{T} 2 * 33$. Little direct prospective information about its relationship to variables in blood transfusion regimens exists, however several lines of evidence suggest a relationship. The first comes from the lack of cardiosiderosis in heavily iron overloaded NTDT patients ${ }^{20,21}$. The next comes from surprising disparity in the frequency of cardiosiderosis between Middle-Eastern and European countries. Baseline data from 11 countries of 925 patients, mainly with TDT $(>99 \%)^{35}$ found the frequency of cardiosiderosis $(\mathrm{T} 2 *<20 \mathrm{~ms})$ was notably lower in the Middle East $(28.5 \%)$ than Europe $(49.5 \%)$ or Asia-Pacific $(40.9 \%)^{35}$. This was despite the proportion of patients with LIC values $>15 \mathrm{mg} / \mathrm{g}$ dry weight being higher in the Middle East $(63 \%)$ than Europe $(51 \%)$. Similar observations have been reported in Egyptian patients ${ }^{36}$. The reason is not immediately obvious: genotypic regional differences in thalassemia mutations are unlikely to explain these findings as systematic differences in frequency of abnormal myocardial T2* have not been noted in migrants from these countries to Europe. In 
Oman, incidences of abnormal T2* are high (46\%) compared to elsewhere in the Middle-East which is more likely to be due to difference in clinical management than to genetic differences from elsewhere in the Middle-East Region as the genotypes are highly heterogeneous ${ }^{37}$.

Could differences in chelation policy account for the low prevalence of abnormal myocardial T2* in the Middle East? Again, this is unlikely, Middle-Eastern patients were generally poorly chelated, with high LIC and ferritin, and indeed many patients had received little or no chelation, and had spent highest proportions of their lives without any chelation than other regional groups ${ }^{35}$. Unfortunately, the pre-transfusion $\mathrm{Hb}$ values were not given. However, the proportion of patients going more than four weeks between transfusions was highest in the Middle-Eastern patients (13\%) compared with Europe $(2.3 \%)$ or Asia-Pacific (5.5\%), transfusion episodes being lowest in the Middle East. In Europe, transfusion regimens close to TIF guidelines are generally followed, with pre-transfusion $\mathrm{Hb}$ values of $9.5-10.5 \mathrm{~g} / \mathrm{dl}$ which would cause a significant suppression of endogenous erythropoiesis using the model of Cazzola ${ }^{5}$. By contrast, reports from Egypt, for example, show pre-transfusion $\mathrm{Hb}$ values of $7.5 \mathrm{~g} / \mathrm{dl}$ or less. In another study, with over 1700 patients, an analysis of transfusion and chelation policy by region (EPIC study) ${ }^{38}$ showed lower annual volume of blood transfused in the Middle East than in Europe or Asia-Pacific.

\section{c) Differences in the activity of erythron with transfusion as marked by soluble transferrin receptors}

The possibility that transfusion policy may impact on cardiosiderosis is strengthened by a recent study where the suppression of the erythron, as marked by sTfR, when combined with a high transfusioniron loading rate was the greatest predictor of cardiosiderosis ${ }^{26}$ (Figure 2). In this study, a large number of risk factors for cardiosiderosis were examined in 73 patients. sTfR levels were significantly lower in patients with cardiosiderosis (odds ratio 21) and this risk increased further when the transfusion-iron loading was taken into account. When rates exceeded the erythroid transferrin uptake rate (derived from sTfR 1$)^{26}$ by $>0.21 \mathrm{mg} / \mathrm{kg} / \mathrm{day}$, the odds ratio increased to 48 . High levels of LIC, ferritin and LPI were also risk factors but less strongly. High levels of bilirubin, reticulocyte counts or low hepcidin were associated with lower risk. A unifying mechanism for these risks, as discussed above, is that unsaturated transferrin is generated by an active bone marrow which in turn removes forms of NTBI such as LPI that would otherwise be taken into myocardium. This suggests transfusion policy and, in particular, the average $\mathrm{Hb}$ levels, estimated by pre-transfusion $\mathrm{Hb}$, will have a key impact on the cardiosiderotic risk. We suggest that regional differences in risk may relate to differences in transfusion policy.

Interestingly, the sTfR-estimated erythropoietic expansion was also more predicated by the age of onset of transfusion dependence than by subsequent years of transfusion ${ }^{26}$. Other data, comparing 1266 TDT patients across 70 centers, support this, where sTfR levels relate to $\mathrm{EMH}^{39}$ and that where EMH persists despite transfusion, patients do not develop cardiosiderosis ${ }^{40}$. Furthermore, in transfused sickle cell disease, the reduction of reticulocytes to $<5 \%$ predicts future development of cardiosiderosis $^{41}$. This points to a fundamental role for residual erythropoiesis in extra-hepatic hemosiderosis. If residual erythropoiesis is maintained at a reasonable level (sweet spot), this constitutes a safety valve against toxic NTBI species and cardiosiderosis ${ }^{26}$

\section{d) Control of iron retention in tissues through ferroportin-hepcidin interactions}

Recent animal work provides novel insight into consequences of ferroportin/hepcidin interaction for the extra-hepatic complications of TDT $^{42-44}$. This work highlights the importance of cellular iron egress, rather than just uptake, to cardiomyocyte iron retention. The hemochromatotic model, resulting from a ferroportin mutation conferring resistance to hepcidin with unhindered ferroportin patency, causes fatal exocrine pancreatic failure due to iron overload of the acinar cells $\mathrm{s}^{42}$. These never express ferroportin and so, unlike myocardium and endocrine system, are unable to export iron acquired from $\mathrm{NTBI}^{42}$. Hence cardiac-specific knock-out of ferroportin, as confirmed elsewhere, results in cardiosiderosis $^{43,44}$. Thus we predict that this under-recognized balance of NTBI uptake to ferroportinmediated release is potentially important to net cardiosiderosis clinically.

Another mechanism by which a low transfusion regimen in TDT may result in decreased cardiosiderosis is therefore through decrease of hepcidin. We have shown that high plasma hepcidin is related to cardiosiderosis in TDT, although not as strongly as is low sTfR (low ETUR) ${ }^{26}$. We can speculate that a low transfusion regime results in lower hepcidin because of less marrow suppression. 
Increased hepcidin, either post-transfusion ${ }^{45,46}$, or as part of high transfusion regimes, is compounded by the switching off of the erythroid iron uptake (safety valve for $\mathrm{LPI}^{26}$ ) thus subjecting cardiomyocytes to a double impact of high LPI/NTBI exposure and limited cardiac iron exit via hepcidin-controlled ferroportin.

\section{Interaction of blood transfusion and chelatable iron pools}

The structure, mechanisms of iron binding, pharmacokinetics, and clinical effects of the clinically licensed iron chelators, desferrioxamine (DFO), deferiprone (DFP) and deferasirox (DFX), have been described in detail recently by the senior author ${ }^{30,47}$, and will not be repeated here in detail. This section will focus of the interaction of blood transfusion on iron chelation therapy.

\section{a) Interaction of transfusion with chelatable iron pools}

Early studies on DFO showed a relationship between blood transfusion and chelatable iron ${ }^{48}$. When DFO binds iron to form ferrioxamine (FO) the iron is derived from two key pools: firstly, the liver and, secondly, from erythrocyte catabolism by macrophages in spleen, liver and bone marrow. DFO is highly efficient at chelating liver iron because of rapid uptake into the hepatocytes ${ }^{49}$. FO can be eliminated in urine or in bile (feces) but is derived from two different sources. Urinary FO is derived from erythrocyte catabolism whereas fecal FO is mainly derived from hepatic iron. The proportion of endogenous red cells is higher pre- than post-transfusion. This leads to increased peripheral hemolysis as well as more intramedullary IE, which in turn increases the magnitude of the chelatable iron pool from red cell catabolism. This iron pool is excreted in urine. Thus pre-transfusion, at low Hb values, urinary iron excretion of FO, derived from erythrocyte catabolism, increases whereas fecal iron excretion is relatively unaffected or decreases slightly.

Factors affecting chelatable iron pools with DFP are less fully described than with DFO and even less is known about the effect of the transfusional iron-loading rate. However we have examined iron pools that are chelatable with DFP by measuring concentrations of the DFP iron complex in plasma at the same time as measuring 24-hour urine iron excretion ${ }^{50}$. Chelated iron in the plasma compartment (forms ferriprone, FP, which is detected using the NTBI assay ${ }^{50}$, Figure 3C) was greatest in patients with the highest LIC levels, as might be expected, but also greater in those with lowest transfusional iron-loading rate (Figure 3A, 3B), where hemolysis and/or IE are greater. Increments of plasma FP also correlate with total urine iron excretion (UIE) and with decrements in LIC over the next year (Figure 3D, 3E). Thus blood transfusion decreases the iron available for chelation by DFP in the plasma compartment by decreasing IE and hemolysis. Conversely, high blood transfusion rate increases total body iron, which in turn increases the LIC-derived pool available for chelation (Figure 3A).

\section{b) Interaction of blood transfusion with chelator dose needed for iron balance}

Iron balance is simply the difference between iron input from gastrointestinal plus transfusional and iron output from chelation. From first principles, the dose of chelation needed under conditions of low transfusion would be better than at high transfusion rates. Body iron can be calculated from LIC using the Angelucci formula ${ }^{13}$ and hence change in LIC can be used to calculate changes in body iron over time.

The interaction of transfusion rate with total iron excretion has been described using this approach for both DFO and DFX. With DFO, at typical transfusional iron loading rates $(0.3-0.5 \mathrm{mg} / \mathrm{kg} / \mathrm{d})$, negative balance was achieved in $75 \%$ of patients prescribed subcutaneously $35-49 \mathrm{mg} / \mathrm{kg} / \mathrm{d} 5$ days $/$ week, whereas at doses of $50 \mathrm{mg} / \mathrm{kg} / \mathrm{d}$ or greater, response rates increased to $86 \%$. At higher iron loading rates $(>0.5 \mathrm{mg} / \mathrm{kg} / \mathrm{d})$, response was seen in only half of the patients prescribed subcutaneously 35 $49 \mathrm{mg} / \mathrm{kg} / \mathrm{d} 5$ days/week, but by increasing this dose to $50 \mathrm{mg} / \mathrm{kg}$ or greater response increased to $89 \%$. With DFX, the importance of the blood transfusion rate to iron balance has also been shown using this approach. At low transfusion rates $<0.3 \mathrm{mg} / \mathrm{kg} / \mathrm{d}, 96 \%$ of patients have a negative iron balance at $30 \mathrm{mg} / \mathrm{kg} / \mathrm{d}$ whereas at higher transfusion rates, only $82 \%{ }^{17}$. The principle of adjusting dose to transfusional loading rate has been built into drug labeling and into the design of further studies of efficacy where serum ferritin was used to adjust dose even though this is a less precise marker of iron balance than LIC. 
With DFP, data on the effects of transfusion rate on chelation efficacy is not so clear from prospective trials but useful data are available from retrospective analysis of LIC trends using SQUID (Superconducting Quantum Interference Device biomagnetometry) in $54 \beta$-thalassaemia major patients receiving DFP $(75 \mathrm{mg} / \mathrm{kg} / \mathrm{d})$ or 51 patients receiving $\mathrm{DFO}^{51}$. Detailed contour plots of the interaction of transfusion with chelation dose were undertaken for both drugs. At $30 \mathrm{mg} / \mathrm{kg} / \mathrm{d}$ of DFO (a low dose), negative iron balance was achieved when transfusion rates were less that $22 \mathrm{mg} /$ day for total body iron stores exceeding $4 \mathrm{~g}$ or when transfusion rates were less than $17 \mathrm{mg} / \mathrm{day}$ for total body iron stores exceeding $1 \mathrm{~g}$. With DFP at $75 \mathrm{mg} / \mathrm{kg} / \mathrm{d}$, negative iron balance was achieved when transfusion rates were less than $17 \mathrm{mg}$ of iron/day for total body iron stores exceeding $1.8 \mathrm{~g}$. Higher doses of both chelators could achieve negative iron balance when the transfusion rate was more than described. In conclusion, the successful dose for balancing input and output of iron of each form of monotherapy is critically dependent of the blood transfusion rate in the treatment group.

\section{Other factors affecting extra-hepatic iron removal with chelation therapy}

The authors do not regard blood transfusion as the sole factor affecting iron removal from extrahepatic tissues; simply that this factor may have been overlooked to some extent. Other factors are clearly important. For removing myocardial iron, which can be achieved with all chelators used alone or in combination ${ }^{30}$, removal of liver iron generally occurs at a much faster rate than from the heart, as shown elegantly by trajectory trends for myocardial and liver iron assessed by serial $\mathrm{MRI}^{34}$. Removing iron from the liver improves removal of myocardial iron: for example in a subsidiary EPIC study on DFX, improvement of cardiac T2* was progressively better from 12 to 36 months in patients who achieved LIC values $<7 \mathrm{mg} / \mathrm{g}$ dry weight than those with LIC values $>15 \mathrm{mg} / \mathrm{g}$ dry weight ${ }^{52}$. Baseline, or pre-treatment, LIC values also seem to impact on success of removing cardiosiderosis. Thus in the 'Cordelia' DFX study, the \% improvement in cardiac T2* with DFX at 1y was greater $(17 \%)$ in patients with baseline LIC $<7 \mathrm{mg} / \mathrm{g}$ compared to those with baseline LIC $>15 \mathrm{mg} / \mathrm{g}$ dry weight $(9 \%$ improvement) ${ }^{53}$ and this pattern was sustained at 2 years ${ }^{54}$.

Regimes where there is 24-hour presence of a chelator in plasma are preferable, as they provide continuous protection from NTBI or LPI uptake since these rebound rapidly after stopping treatment $^{55,56}$. However, not all forms of NTBI, available for uptake into cardiomyocytes, are equally inhibited $^{26}$. The rate of access of the free chelator ligand and the rate of egress of the iron complex, determined by the size, charge, and lipid solubility ${ }^{57}$, are important to their ability to chelate intracellular iron pools. DFP, by virtue of its neutral charge and low molecular weight, has faster kinetic access to intracellular iron pools than $\mathrm{DFO}^{58}$. The interpretation of clinical studies however is somewhat problematic because randomized head-to-head comparison of DFP with DFO have used suboptimal low doses of DFO but relatively high doses of $\mathrm{DFP}^{59,60}$ and baseline LIC values often vary considerably between studies. The issue of combination of chelators providing a shuttling mechanism to enhance chelation rates is theoretically justified ${ }^{61-63}$, but the extent to which shuttling contributes to enhanced chelation, if the drugs are not given simultaneously, is questionable. Clinical benefit from sequential combinations is more likely to be a consequence of greater overall exposure to chelation, contributed to by improved adherence.

\section{Figure legends}

Figure 1. Factors influencing the generation of NTBI. (adapted from Porter et al 2014, BJH)

Figure 2. Statistically significant risk factors for cardiosiderosis in TDT.

In 73 patients ( 24 with and 49 without cardiosiderosis) various relevant factors were assessed (after Garbowski et al 2017, Haematologica) for a relationship with cardiosiderosis. Thresholds protecting from cardiosiderosis are shown. ROC curves were constructed for every variable and a threshold protecting from cardiosiderosis for highest likelihood ratio was reported. Strength of association was expressed using an odds ratio. Factors not associated with cardiosiderosis were NRBC, total serum iron, weight, ILR, transferrin saturation, GDF-15, NTBI, years of transfusion dependence. LPI, labiile plasma iron; abs retic, absolute reticulocyte count; ETU erythroid transferrin uptake; sTfR, soluble transferrin receptor 1; ETUR, erythroid transferrin uptake rate; ILR, iron load rate

Figure 3. The effect of transfusion and iron stores on chelatable iron pools. 
Relationship of the 1-week change in NTBI after commencing deferiprone therapy to baseline LIC (A) and the reciprocal of transfusion iron-loading rate, 1/TIRL (B). 1-week change in NTBI correlates with plasma ferriprone levels (C), with urinary iron excretion (D) and with change in LIC after 1 year (E). Patients on DFP monotherapy (circles) and DFP with DFO (triangles). Adapted from Aydinok et al 2012, Haematologica.

\section{References}

1. Gabutti V, Piga A, Fortina P, Miniero R, Nicola P. Correlation between transfusion requirement, blood volume and haemoglobin level in homozygous beta-thalassaemia. Acta Haematol. 1980;64(2):103-108.

2. Gabutti V, Piga A, Nicola P, et al. Haemoglobin levels and blood requirement in thalassaemia. Arch Dis Child. 1982;57(2):156-157.

3. Brunengo MA, Girot R. [Transfusion requirements and mean annual hemoglobin level in thalassemia major]. Nouv Rev Fr Hematol. 1986;28(5):309-313.

4. Rebulla P, Modell B. Transfusion requirements and effects in patients with thalassaemia major. Cooleycare Programme. Lancet. 1991;337(8736):277-80.

5. Cazzola M, Borgna-Pignatti C, Locatelli F, Ponchio L, Beguin Y, De Stefano P. A moderate transfusion regimen may reduce iron loading in beta-thalassemia major without producing excessive expansion of erythropoiesis. Transfusion. 1997;37(2):135-40. doi:10.1046/j.15372995.1997.37297203514.x.

6. Cappellini M-D, Cohen A, Porter J (Hematologist), Taher A, Viprakasit V, Thalassaemia International Federation. Guidelines for the management of transfusion dependent thalassaemia (TDT).

7. Ragab LA, Hamdy MM, Shaheen IA, Yassin RN. Blood transfusion among thalassemia patients: A single Egyptian center experience. Asian J Transfus Sci. 2013;7(1):33-6. doi:10.4103/0973-6247.106728.

8. Premawardhena A, Fisher CA, Olivieri NF, et al. Haemoglobin E $\beta$ thalassaemia in Sri Lanka. Lancet. 2005;366(9495):1467-1470. doi:10.1016/S0140-6736(05)67396-5.

9. Porter JB, Cappellini MD, Kattamis A, et al. Iron overload across the spectrum of nontransfusion-dependent thalassaemias: role of erythropoiesis, splenectomy and transfusions. $\mathrm{Br} J$ Haematol. 2016. doi:10.1111/bjh.14373.

10. Pippard MJ, Callender ST, Warner GT, Weatherall DJ. Iron absorption and loading in $\beta$ thalassaemia intermedia. Lancet. 1979;2(8147):819-821.

11. Pootrakul P, Kitcharoen K, Yansukon P, et al. The effect of erythroid hyperplasia on iron balance. Blood. 1988;71(4):1124-9.

12. Taher AT, Porter J, Viprakasit V, et al. Deferasirox reduces iron overload significantly in nontransfusion-dependent thalassemia: 1-Year results from a prospective, randomized, doubleblind, placebo-controlled study. Blood. 2012;120(5):970-977. doi:10.1182/blood-2012-02412692.

13. Angelucci E, Brittenham GM, McLaren CE, et al. Hepatic iron concentration and total body iron stores in thalassemia major. N Engl J Med. 2000;343(5):327-31. doi:10.1056/NEJM200008033430503.

14. Tanno T, Bhanu N V, Oneal P a, et al. High levels of GDF15 in thalassemia suppress expression of the iron regulatory protein hepcidin. Nat Med. 2007;13(9):1096-1101. doi:10.1038/nm1629.

15. Kautz L, Jung G, Valore E V, Rivella S, Nemeth E, Ganz T. Identification of erythroferrone as an erythroid regulator of iron metabolism. Nat Genet. 2014;46(7):678-684. doi:10.1038/ng.2996.

16. Dussiot M, Maciel TT, Fricot A, et al. An activin receptor IIA ligand trap corrects ineffective erythropoiesis in $\beta$-thalassemia. Nat Med. 2014;20(4):398-407. doi:10.1038/nm.3468.

17. Cohen AR, Glimm E, Porter JB. Effect of transfusional iron intake on response to chelation therapy in $\beta$-thalassemia major. Blood. 2008;111(2):583-587. doi:10.1182/blood-2007-08109306.

18. O'Brien RT, Pearson HA, Spencer RP. Transfusion-induced decrease in spleen size in thalassemia major: documentation by radioisotopic scan. J Pediatr. 1972;81(1):105-107.

19. Origa R, Galanello R, Ganz T, et al. Liver iron concentrations and urinary hepcidin in betathalassemia. Haematologica. 2007;92(5):583-588. doi:10.3324/haematol.10842.

20. Taher AT, Musallam KM, Wood JC, Cappellini MD. Magnetic resonance evaluation of hepatic and myocardial iron deposition in transfusion-independent thalassemia intermedia compared to 
regularly transfused thalassemia major patients. Am J Hematol. 2010;85(4):288-290. doi:10.1002/ajh.21626.

21. Roghi A, Cappellini MD, Wood JC, et al. Absence of cardiac siderosis despite hepatic iron overload in Italian patients with thalassemia intermedia: an MRI T2* study. Ann Hematol. 2010;89(6):585-9. doi:10.1007/s00277-009-0879-3.

22. Oudit GY, Sun H, Trivieri MG, et al. L-type Ca2+ channels provide a major pathway for iron entry into cardiomyocytes in iron-overload cardiomyopathy. Nat Med. 2003;9(9):1187-1194. doi: $10.1038 / \mathrm{nm} 920$.

23. Pinilla-Tenas JJ, Sparkman BK, Shawki A, et al. Zip14 is a complex broad-scope metal-ion transporter whose functional properties support roles in the cellular uptake of zinc and nontransferrin-bound iron.

24. Porter JB, Walter PB, Neumayr LD, et al. Mechanisms of plasma non-transferrin bound iron generation: Insights from comparing transfused diamond blackfan anaemia with sickle cell and thalassaemia patients. Br J Haematol. 2014;167(5):692-696. doi:10.1111/bjh.13081.

25. Bradley SJ, Gosriwitana I, Srichairatanakool S, Hider RC, Porter JB. Non-transferrin-bound iron induced by myeloablative chemotherapy. Br J Haematol. 1997;99(2):337-43.

26. Garbowski MW, Evans P, Vlachodimitropoulou E, Hider R, Porter JB. Residual erythropoiesis protects against myocardial hemosiderosis in transfusion-dependent thalassemia by lowering labile plasma iron via transient generation of apotransferrin. Haematologica. 2017:haematol.2017.170605. doi:10.3324/haematol.2017.170605.

27. Evans RW, Rafique R, Zarea A, et al. Nature of non-transferrin-bound iron: Studies on iron citrate complexes and thalassemic sera. J Biol Inorg Chem. 2008;13(1):57-74. doi:10.1007/s00775-007-0297-8.

28. Esposito BP, Breuer W, Sirankapracha P, Pootrakul P, Hershko C, Cabantchik ZI. Labile plasma iron in iron overload: Redox activity and susceptibility to chelation. Blood. 2003;102(7):2670-2677. doi:10.1182/blood-2003-03-0807.

29. Porter JB, de Witte T, Cappellini MD, Gattermann N. New insights into transfusion-related iron toxicity: Implications for the oncologist. Crit Rev Oncol Hematol. 2016;99:261-271. doi:10.1016/j.critrevonc.2015.11.017.

30. Porter JB. Treatment of Systemic Iron Overload. In: Crichton RR, Ward RJ, Hider RC, eds. Metal Chelation in Medicine. Royal Society of Chemistry; 2016:106-152.

31. Buja LM, Roberts WC. Iron in the heart. Etiology and clinical significance. Am J Med. 1971;51(2):209-221. doi:10.1016/0002-9343(71)90240-3.

32. Jensen PD, Jensen FT, Christensen T, Nielsen JL, Ellegaard J. Relationship between hepatocellular injury and transfusional iron overload prior to and during iron chelation with desferrioxamine: A study in adult patients with acquired anemias. Blood. 2003;101(1):91-96. doi:10.1182/blood-2002-06-1704.

33. Anderson LJ, Holden S, Davis B, et al. Cardiovascular T2-star (T2*) magnetic resonance for the early diagnosis of myocardial iron overload. Eur Heart J. 2001;22(23):2171-2179. doi:10.1053/euhj.2001.2822.

34. Noetzli LJ, Carson SM, Nord AS, Coates TD, Wood JC. Longitudinal analysis of heart and liver iron in thalassemia major. Blood. 2008;112(7):2973-2978.

35. Aydinok Y, Porter JB, Piga A, et al. Prevalence and distribution of iron overload in patients with transfusion-dependent anemias differs across geographic regions: Results from the CORDELIA study. Eur J Haematol. 2015;95(3):244-253. doi:10.1111/ejh.12487.

36. El Beshlawy A, El Tagui M, Hamdy M, et al. Low prevalence of cardiac siderosis in heavily iron loaded Egyptian thalassemia major patients. Ann Hematol. 2014;93(3):375-379. doi:10.1007/s00277-013-1876-0.

37. Hassan SM, Harteveld CL, Bakker E, Giordano PC. Broader Spectrum of $\boldsymbol{\beta}$-Thalassemia Mutations in Oman: Regional Distribution and Comparison with Neighboring Countries. Hemoglobin . 2015;39(2):107-110. doi:10.3109/03630269.2015.1009632.

38. Viprakasit V, Gattermann N, Lee JW, et al. Geographical variations in current clinical practice on transfusions and iron chelation therapy across various transfusion-dependent anaemias. Blood Transfus. 2013;11(1):108-122. doi:10.2450/2012.0012-12.

39. Ricchi P, Ammirabile M, Costantini S, et al. A useful relationship between the presence of extramedullary erythropoeisis and the level of the soluble form of the transferrin receptor in a large cohort of adult patients with thalassemia intermedia: A prospective study. Ann Hematol. 2012;91(6):905-909. doi:10.1007/s00277-011-1385-y.

40. Ricchi P, Meloni A, Spasiano A, et al. Extramedullary hematopoiesis is associated with lower cardiac iron loading in chronically transfused thalassemia patients. Am J Hematol. 
2015;90(11):1008-1012. doi:10.1002/ajh.24139.

41. Meloni A, Puliyel M, Pepe A, Berdoukas V, Coates TD, Wood JC. Cardiac iron overload in sickle-cell disease. Am J Hematol. 2014;89(7):678-683. doi:10.1002/ajh.23721.

42. Altamura S, Kessler R, Groene HJ, et al. Resistance of ferroportin to hepcidin binding causes exocrine pancreatic failure and fatal iron overload. Cell Metab. 2014;20(2):359-367. doi:10.1016/j.cmet.2014.07.007.

43. Lakhal-Littleton S, Wolna M, Carr CA, et al. Cardiac ferroportin regulates cellular iron homeostasis and is important for cardiac function. Proc Natl Acad Sci U S A. 2015;112(10):3164-3169. doi:10.1073/pnas.1422373112.

44. Lakhal-Littleton S, Wolna M, Chung YJ, et al. An essential cell-autonomous role for hepcidin in cardiac iron homeostasis. Elife. 2016;5(NOVEMBER2016). doi:10.7554/eLife.19804.

45. Jones E, Pasricha SR, Allen A, et al. Hepcidin is suppressed by erythropoiesis in hemoglobin e $\beta$-thalassemia and $\beta$-thalassemia trait. Blood. 2015;125(5):873-880. doi:10.1182/blood-201410-606491.

46. Pasricha SR, Frazer DM, Bowden DK, Anderson GJ. Transfusion suppresses erythropoiesis and increases hepcidin in adult patients with $\beta$-thalassemia major: A longitudinal study. Blood. 2013;122(1):124-133. doi:10.1182/blood-2012-12-471441.

47. Porter J, Hershko C. The Properties of Clinically Useful Iron Chelators. In: Anderson HJ, McLaren GD, eds. Iron Physiology and Pathophysiology in Humans. Humana Press; 2012:591-630.

48. Pippard MJ, Callender ST, Finch C a. Ferrioxamine excretion in iron-loaded man. Blood. 1982;60(2):288-94.

49. Porter JB, Rafique R, Srichairatanakool S, et al. Recent insights into interactions of deferoxamine with cellular and plasma iron pools: implications for clinical use. Ann NY Acad Sci. 2005;1054:155-168.

50. Aydinok Y, Evans P, Manz CY, Porter JB. Timed non-transferrin bound iron determinations probe the origin of chelatable iron pools during deferiprone regimens and predict chelation response. Haematologica. 2012;97(6):835-841. doi:10.3324/haematol.2011.056317.

51. Fischer R, Longo F, Nielsen P, Engelhardt R, Hider RC, Piga A. Monitoring long-term efficacy of iron chelation therapy by deferiprone and desferrioxamine in patients with beta-thalassaemia major: application of SQUID biomagnetic liver susceptometry. Br J Haematol. 2003;121(6):938-948. doi:10.1046/j.1365-2141.2003.04297.x.

52. Porter J, Taher AT, Aydinok Y, et al. Impact Of Liver Iron Overload On Myocardial T2* Response In Transfusion-Dependent Thalassemia Major Patients Treated With Deferasirox For Up To 3 Years. Blood (ASH Annu Meet Abstr. 2013;122(21):1016.

53. Pennell DJ, Porter JB, Piga A, et al. A 1-year randomized controlled trial of deferasirox vs deferoxamine for myocardial iron removal in -thalassemia major (CORDELIA). Blood. 2014;123(10):1447-1454. doi:10.1182/blood-2013-04-497842.

54. Pennell DJ, Porter JB, Piga A, et al. Sustained improvements in myocardial T2* over 2 years in severely iron-overloaded patients with beta thalassemia major treated with deferasirox or deferoxamine. Am J Hematol. 2015;90(2):91-96. doi:10.1002/ajh.23876.

55. Porter JB, Abeysinghe RD, Marshall L, Hider RC, Singh S. Kinetics of removal and reappearance of non-transferrin-bound plasma iron with deferoxamine therapy. Blood. 1996;88(2):705-13.

56. Cabantchik ZI, Breuer W, Zanninelli G, Cianciulli P. LPI-labile plasma iron in iron overload. Best Pract Res Clin Haematol. 2005;18(2 SPEC. ISS.):277-287. doi:10.1016/j.beha.2004.10.003.

57. Porter JB, Gyparaki M, Burke LC, et al. Iron mobilization from hepatocyte monolayer cultures by chelators: The importance of membrane permeability and the iron-binding constant. Blood. 1988;72(5):1497-1503.

58. Cooper CE, Lynagh GR, Hoyes KP, Hider RC, Cammack R, Porter JB. The relationship of intracellular iron chelation to the inhibition and regeneration of human ribonucleotide reductase. J Biol Chem. 1996;271(34):20291-20299. doi:10.1074/jbc.271.34.20291.

59. Tanner MA, Galanello R, Dessi C, et al. A randomized, placebo-controlled, double-blind trial of the effect of combined therapy with deferoxamine and deferiprone on myocardial iron in thalassemia major using cardiovascular magnetic resonance. Circulation. 2007;115(14):18761884. doi:10.1161/CIRCULATIONAHA.106.648790.

60. Pennell DJ. Randomized controlled trial of deferiprone or deferoxamine in beta-thalassemia major patients with asymptomatic myocardial siderosis. Blood. 2006;107(9):3738-3744.

doi:10.1182/blood-2005-07-2948. 
61. Evans P, Kayyali R, Hider RC, Eccleston J, Porter JB. Mechanisms for the shuttling of plasma non-transferrin-bound iron (NTBI) onto deferoxamine by deferiprone. Transl Res. 2010;156(2):55-67. doi:10.1016/j.trs1.2010.05.002.

62. Vlachodimitropoulou Koumoutsea E, Garbowski M, Porter J. Synergistic intracellular iron chelation combinations: Mechanisms and conditions for optimizing iron mobilization. $\mathrm{Br} J$ Haematol. 2015;170(6):874-883. doi:10.1111/bjh.13512.

63. Vlachodimitropoulou E, Chen Y-L, Garbowski M, et al. Eltrombopag: a powerful chelator of cellular or extracellular iron(III) alone or combined with a second chelator. Blood. 2017;130(17):1923-1933. doi:10.1182/blood-2016-10-740241. 